\title{
Study Site Temporarily Closed to Accrual and Intervention
}

National Cancer Institute

\section{Source}

National Cancer Institute. Study Site Temporarily Closed to Accrual and Intervention. NCI

Thesaurus. Code C126839.

Trial is temporarily not accruing and patients are not receiving therapy/intervention. 